Research Article

\title{
Network Pharmacology Strategy to Investigate the Pharmacological Mechanism of Siwu Decoction on Primary Dysmenorrhea and Molecular Docking Verification
}

\author{
Dandan Jiang $\mathbb{D}^{1},{ }^{1}$ Xiaoyan Wang $\mathbb{D},{ }^{1}$ Lijun Tian $\mathbb{D},{ }^{2}$ and Yufeng Zhang $\mathbb{D}^{3}$ \\ ${ }^{1}$ Department of Gynaecology and Obstetrics, Nantong Third People's Hospital, Nantong University, Nantong, \\ Jiangsu 226001, China \\ ${ }^{2}$ Department of Critical Care Medicine, Nantong Third People's Hospital, Nantong University, Nantong, Jiangsu 226001, China \\ ${ }^{3}$ Department of Respiratory Medicine, Jiangyin Hospital of Traditional Chinese Medicine, \\ Jiangyin Hospital Affiliated to Nanjing University of Chinese Medicine, Jiangyin, Jiangsu 214400, China \\ Correspondence should be addressed to Yufeng Zhang; yufengzhang@njucm.edu.cn
}

Received 2 November 2020; Revised 26 November 2020; Accepted 29 January 2021; Published 15 February 2021

Academic Editor: Linus R. Shao

Copyright ( $\odot 2021$ Dandan Jiang et al. This is an open access article distributed under the Creative Commons Attribution License, which permits unrestricted use, distribution, and reproduction in any medium, provided the original work is properly cited.

\begin{abstract}
Objective. To study the pharmacological mechanisms of Siwu decoction (SWD) on primary dysmenorrhea (PDM) and verify with molecular docking. Methods. The Traditional Chinese Medicine Systems Pharmacology Database and Analysis Platform (TCMSP) was utilized to acquire the active compounds and their corresponding target genes. The GeneCards database was utilized in the search for target genes that were associated with PDM. The intersection genes from the active target genes of SWD and those associated with PDM represented the active target genes of SWD that act on PDM. The Gene Ontology (GO) function enrichment and Kyoto Encyclopedia of Genes and Genomes (KEGG) pathway enrichment analyses were both carried out by RGUI 3.6.1 and Cytoscape 3.6.0 software. Cytoscape was also utilized for creating a compound-target network, and a proteinprotein interaction (PPI) network was created through the STRING database. Molecular docking simulations of the macromolecular protein target receptors and their corresponding compounds were performed using AutoDockTool 1.5.6 and AutoDock Vina software. Results. We identified 14 active compounds as well as 97 active target genes of SWD by using the TCMSP. We compared the 97 active target genes of SWD to the 299 target genes related to PDM, and 23 active target genes for SWD that act on PDM which correlated with 11 active compounds were detected. The compound-target network as well as the PPI network were created, in addition to selecting the most essential compounds and their targets in order to create a key compoundtarget network. The most essential compounds were kaempferol, beta-sitosterol, stigmasterol, and myricanone. The key targets were AKT1, PTGS2, ESR1, AHR, CASP3, and PGR. Lastly, molecular docking was used to confirm binding of the target with its corresponding compound. Conclusion. The pharmacological mechanisms of SWD that act on PDM were investigated, and the active compounds in the SWD for treating PDM were further verified.
\end{abstract}

\section{Introduction}

Primary dysmenorrhea (PDM) is defined as dysmenorrhea resulting from nonpelvic organic lesions [1]. The clinical manifestations of PDM include lower abdominal spasmodic pain during menstrual periods accompanied by headache, nausea, vomiting, and lumbar and leg pain [2].

Oral contraceptives and nonsteroidal anti-inflammatory drugs (NSAIDs) have been prescribed as first choice of treatment in PDM [3, 4]. However, these drugs have adverse effects, and their long-term effects are less than optimal. Lately, there have been studies in which Chinese herbal medicine (CHM) was given as PDM therapy, and the results showed that certain curative effect was achieved [5-7].

The Siwu decoction (SWD), first used during the Tang Dynasty as the basic prescription and the first prescription in the treatment of gynecological diseases, primarily consists of baishao, chuanxiong, danggui, and shudihuang, with the 
Latin names Radix Paeoniae Alba (RPA), Rhizoma Chuanxiong (RC), Radix Angelicae Sinensis (RAS), and Radix Rehmanniae Preparata (RRP), respectively $[8,9]$.

SWD is used for tonifying blood, activating blood, regulation of menstruation, and alleviating pain. A metaanalysis and systematic review reported the effects of SWD as treatment for PDM where beneficial and potentially safer in comparison to standard therapy [10].

In the present study, a network pharmacology approach was used to identify the pharmacological mechanism of SWD on PDM; in addition, we performed molecular docking for verification. We first identified both the active compounds of the four herbs as well as the target genes in SWD. Then, the active target genes of SWD and the target genes related to PDM were used to detect the active target genes of SWD that act on PDM. Gene Ontology (GO) function enrichment and Kyoto Encyclopedia of Genes and Genomes (KEGG) pathway enrichment were both carried out. Subsequently, we constructed the compound-target network and the protein-protein interaction (PPI) network, and the most essential compounds and targets were screen out. Finally, we used these to create a key compound-target network, followed by performing molecular docking verification. Figure 1 illustrates the flowchart of our study.

\section{Materials and Methods}

2.1. Screening the Active Compounds in SWD. Data on the compounds that can be found in the four herbs (RPA, RC, RAS, and RRP) were acquired from the Traditional Chinese Medicine Systems Pharmacology Database and Analysis Platform (TCMSP) (http://tcmspw.com/tcmsp.php) [11]. TCMSP is considered as a one-of-a-kind platform of systems pharmacology of CHM that includes details regarding the association between different diseases, medicine, and their targets. The oral bioavailability $(\mathrm{OB})$ was preset at $\geq 30 \%$, in addition to druglikeness (DL) at $\geq 0.18$, as filter benchmarks during the screening for compounds regarded as active. OB indicates the corresponding quantity and pace at which the body absorbs the drugs into the bloodstream. DL is defined as the equivalence between compounds and previously identified drugs [12].

2.2. Screening the Target Genes of Active Compounds. Data on the genes that are targeted by the active compounds were also retrieved from the TCMSP. In the search format "homo sapiens" was chosen, and we imported the target genes into the Universal Protein Resource (UniProt) knowledge base, an extensive and high-quality online source that contains information regarding annotation data and protein sequences (http://www.uniprot.org/) [13]. Then, the human official gene symbols of these target genes were identified, and they were also considered as the proportion of target genes of SWD that are active.

2.3. Identification of PDM-Related Target Genes and Acquisition of Active Target Genes of SWD with Effects on PDM. "Primary dysmenorrhea" was the key word that was used for searching the GeneCards database (https://www.genecards. org/) [14], a database providing comprehensive data on the predicted and annotated type of human genes. The PDMrelated target genes were searched and acquired. Then, the intersecting genes derived from the active target genes of SWD and the PDM-related target genes were designated as the active target genes of SWD which have effects on PDM.

\subsection{GO Function Enrichment and KEGG Pathway Enrichment} Analysis. The RGUI 3.6.1 as well as org.Hs.eg.db packages were used for retrieval of the entrezIDs of the active target genes. The clusterProfiler package, RGUI, and Cytoscape 3.6.0 software with the plugin apps, CluePedia and ClueGO, were employed to carry out the GO function enrichment analysis as well as analysis of the KEGG pathway enrichment. The GO function enrichment consisted of the analysis of the biological process (BP), molecular function (MF), and cellular component (CC) [15-18].

2.5. Development of the Compound-Target Network and Selection of Key Compounds. Cytoscape software with the incorporated NetworkAnalyzer tool function was used to develop as well as evaluate a network of compounds and targets [16]. The nodes depict the target genes and compounds, while the edges depict the correlations that exist between them. Based on the degree of the relationship between the target genes and compounds, the key compounds in the SWD that acted on PDM were selected.

2.6. Development of PPI Network and Designation of the Key Targets. A PPI network consisting of the SWD that acts on PDM was constructed after introducing the active target genes into the STRING database, which is used for the discovery of functional interactions within genomewide experimental datasets (https://string-db.org/) [19]. We created a PPI network by setting the type of species being researched as "homo sapiens" and the interaction score at a minimum value of 0.4 . Then, we used the PPI network to make a topology analysis, and Cytoscape and its Network Analyzer tool were used to determine the exact degree values of every target gene in order to select the key targets of SWD that act on PDM.

2.7. Verification of Molecular Docking. Molecular docking was utilized in order to confirm the binding of target and its corresponding compound. Data on the construction of the small-molecule compounds was acquired via the PubChem Database (https://pubchem.ncbi.nlm.nih.gov/), and the macromolecular protein target receptors were retrieved via the RCSB PDB database (http://www.rcsb.org/). Molecular docking simulations of macromolecular protein target receptors and their corresponding compounds were performed using AutoDockTool 1.5.6 and AutoDock Vina software [20].

2.8. Statistical Analysis. A part of the statistical analysis was simultaneously conducted with the biotechnology add-ons of the software and platforms that have been disclosed in 


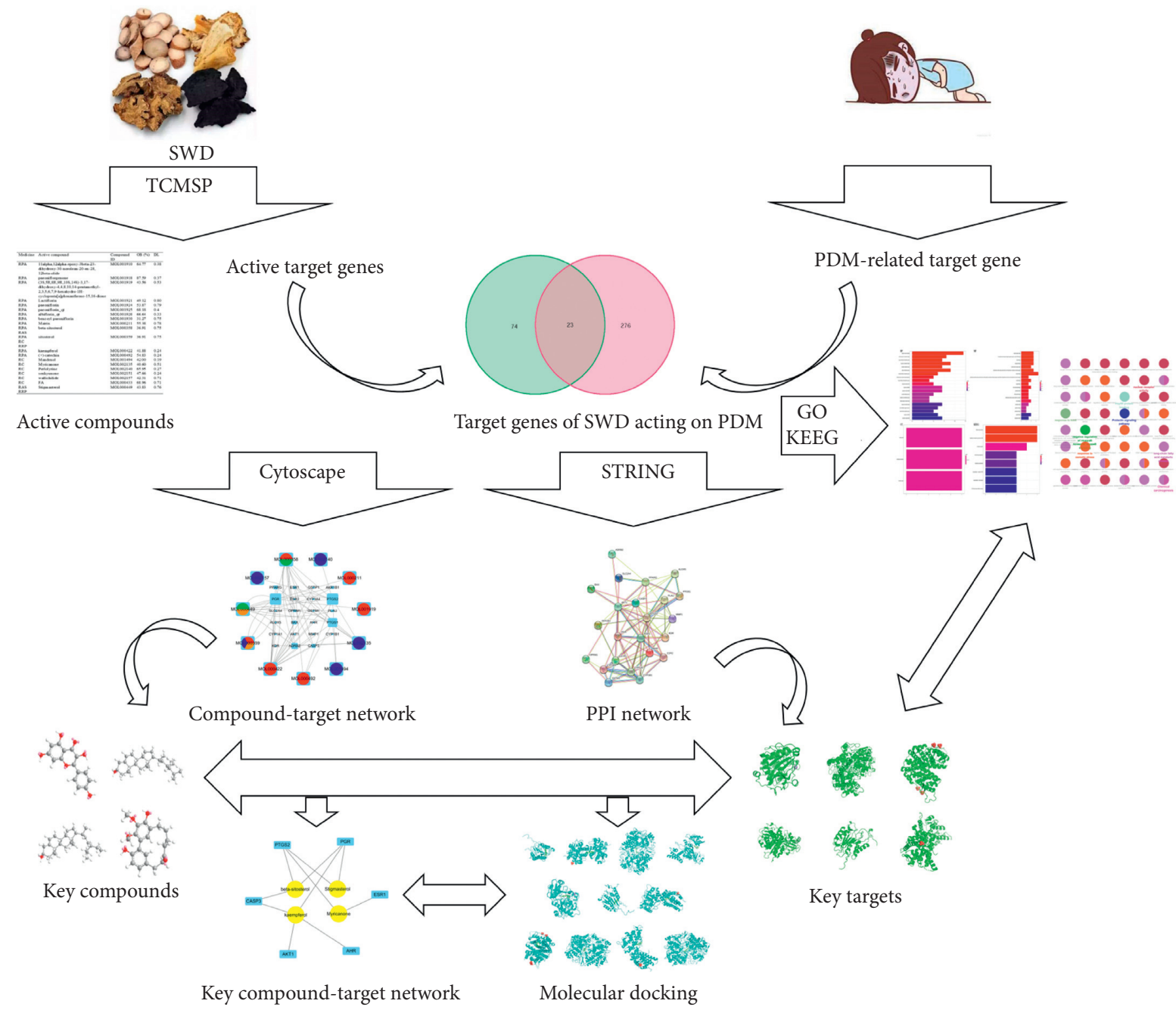

FIgURE 1: Flowchart of the present study.

previous sections. In the GO function and KEGG pathway enrichment, an adjusted P (adj. P) value was used and adj. $P<0.05$ was perceived as statistically significant.

\section{Results}

3.1. Screened Active Compounds of SWD. A total number of 86 compounds were obtained from RPA, 189 compounds from RC, 125 compounds from RAS, and 76 compounds from RRP using the TCMSP (Supplementary Files 1A-1D). By adjusting the filter criteria to $\mathrm{OB} \geq 30 \%$ as well as $\mathrm{DL}$ $\geq 0.18$, the following number of active compounds was selected: 13 from RPA, 7 from RC, 2 from RAS, and 2 from RRP. Lastly, 20 active compounds of SWD were verified with the exclusion of duplications. Table 1 contains the general data on the active compounds in the SWD.

3.2. Screening Active Target Genes of SWD. Data on the target genes related to the 20 active compounds have been retrieved from the TCMSP as well, in which 6 compounds (MOL001910, MOL001921, MOL001925, MOL001928, MOL001930, and MOL002151) did not have targets
(Supplementary File 2A). Then, we screened equivalent symbols depicting genes in the UniProt knowledge base (Supplementary File 2B). Finally, a total of 97 active target genes related to 14 active compounds of SWD were detected (Supplementary File 2C).

\subsection{Attained PDM-Related Target Genes and Identified Active} Target Genes of SWD that Act on PDM. "Primary dysmenorrhea" was the key word that was used to perform a search in the GeneCards database, which resulted in the identification of 299 PDM-related target genes (Supplementary File $3 \mathrm{~A}$ and $3 \mathrm{~B})$.

A comparison was conducted between the previously identified 97 active target genes of SWD and the 299 PDMrelated target genes, leading to the identification of 23 active target genes of SWD that act on PDM (Figure 2 and Table 2).

3.4. The GO Function Enrichment and KEGG Pathway Enrichment Analysis. The entrez IDs of the active target genes of SWD that act on PDM were retrieved through RGUI as well as org.Hs.eg.db (Table 2). Thereafter, we conducted the 
TABLE 1: General data on the active compounds in the SWD.

\begin{tabular}{|c|c|c|c|c|}
\hline Medicine & Active compound & $\begin{array}{l}\text { Compound } \\
\text { ID }\end{array}$ & $\begin{array}{l}\mathrm{OB} \\
(\%)\end{array}$ & $\mathrm{DL}$ \\
\hline RPA & $11 \alpha, 12 \alpha$-Epoxy-3beta-23-dihydroxy-30-norolean-20-en-28, 12beta-olide & MOL001910 & 64.77 & 0.38 \\
\hline RPA & Paeoniflorgenone & MOL001918 & 87.59 & 0.37 \\
\hline RPA & $\begin{array}{c}\text { (3S,5R,8R,9R,10S,14S)-3,17-Dihydroxy-4,4,8,10,14-pentamethyl-2,3,5,6,7,9-hexahydro-1H- } \\
\text { cyclopenta[a]phenanthrene-15,16-dione }\end{array}$ & MOL001919 & 43.56 & 0.53 \\
\hline RPA & Lactiflorin & MOL001921 & 49.12 & 0.80 \\
\hline RPA & Paeoniflorin & MOL001924 & 53.87 & 0.79 \\
\hline RPA & Paeoniflorin_qt & MOL001925 & 68.18 & 0.4 \\
\hline RPA & Albiflorin_qt & MOL001928 & 66.64 & 0.33 \\
\hline RPA & Benzoyl paeoniflorin & MOL001930 & 31.27 & 0.75 \\
\hline RPA & Mairin & MOL000211 & 55.38 & 0.78 \\
\hline RPA & Beta-sitosterol & MOL000358 & 36.91 & 0.75 \\
\hline \multicolumn{5}{|l|}{ RAS } \\
\hline RPA & Sitosterol & MOL000359 & 36.91 & 0.75 \\
\hline \multicolumn{5}{|l|}{$\mathrm{RC}$} \\
\hline \multicolumn{5}{|l|}{ RRP } \\
\hline RPA & Kaempferol & MOL000422 & 41.88 & 0.24 \\
\hline RPA & $(+)$-Catechin & MOL000492 & 54.83 & 0.24 \\
\hline $\mathrm{RC}$ & Mandenol & MOL001494 & 42.00 & 0.19 \\
\hline $\mathrm{RC}$ & Myricanone & MOL002135 & 40.60 & 0.51 \\
\hline $\mathrm{RC}$ & Perlolyrine & MOL002140 & 65.95 & 0.27 \\
\hline $\mathrm{RC}$ & Senkyunone & MOL002151 & 47.66 & 0.24 \\
\hline $\mathrm{RC}$ & Wallichilide & MOL002157 & 42.31 & 0.71 \\
\hline $\mathrm{RC}$ & FA & MOL000433 & 68.96 & 0.71 \\
\hline RAS & Stigmasterol & MOL000449 & 43.83 & 0.76 \\
\hline RRP & & & & \\
\hline
\end{tabular}

Active target genes of SWD

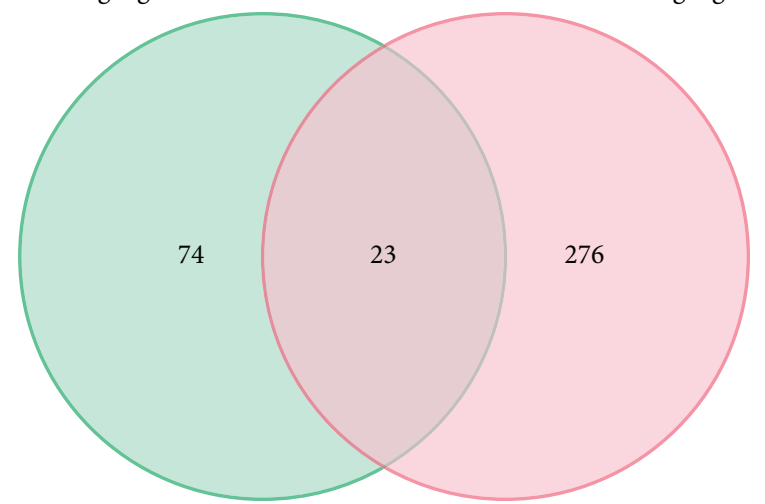

Figure 2: Active target genes of SWD that act on PDM. The 97 active target genes of SWD were compared with the 299 PDMrelated target genes, and 23 active target genes of SWD acting on PDM were identified.

GO function enrichment and KEGG pathway enrichment analyses with the RGUI and clusterProfiler package.

The GO function enrichment analysis of BP indicated the following: active target genes of SWD that act on PDM have been enriched significantly as reaction to toxic substances, the cellular response to xenobiotic stimulus, the metabolic processes of long-chain fatty acids, fatty acids, and unsaturated fatty acids, the response to xenobiotic stimulus, the xenobiotic metabolic process, the maternal process related to female pregnancy, the long-chain fatty acid biosynthetic process, the response to osmotic stress, and other
TABLE 2: Gene symbols and entrezIDs of the active target genes.

\begin{tabular}{lc}
\hline Gene symbol & EntrezID \\
\hline PGR & 5241 \\
PTGS1 & 5742 \\
PTGS2 & 5743 \\
ADRB2 & 154 \\
OPRM1 & 4988 \\
BAX & 581 \\
CASP3 & 836 \\
PPARG & 5468 \\
AKT1 & 207 \\
MMP1 & 4312 \\
CYP3A4 & 1576 \\
CYP1A1 & 1543 \\
CYP1B1 & 1545 \\
ALOX5 & 240 \\
GSTP1 & 2950 \\
AHR & 196 \\
SLC2A4 & 6517 \\
GSTM1 & 2944 \\
ESR1 & 2099 \\
KDR & 3791 \\
ESR2 & 2100 \\
AKR1B1 & 231 \\
PLAU & 5328 \\
\hline
\end{tabular}

processes (Supplementary File 4A). The 20 most enriched GO BP are visualized in Figure 3(a) and ranked according to their adj. $P$ values.

The GO function enrichment analysis of MF indicated the following: the active target genes of SWD that act on 


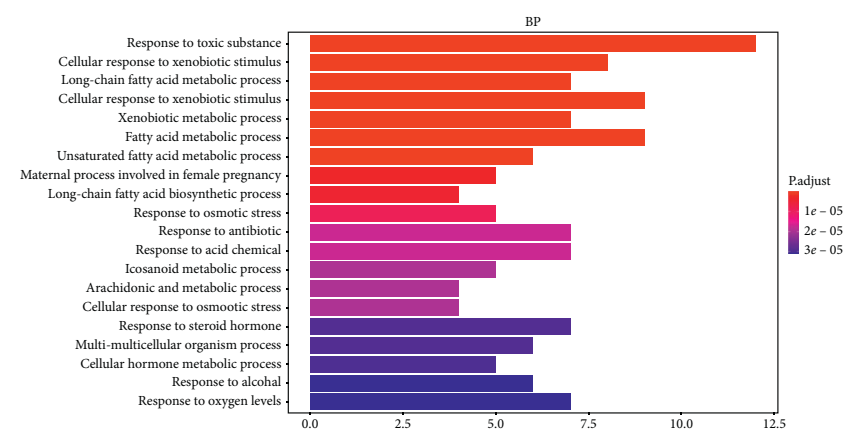

(a)

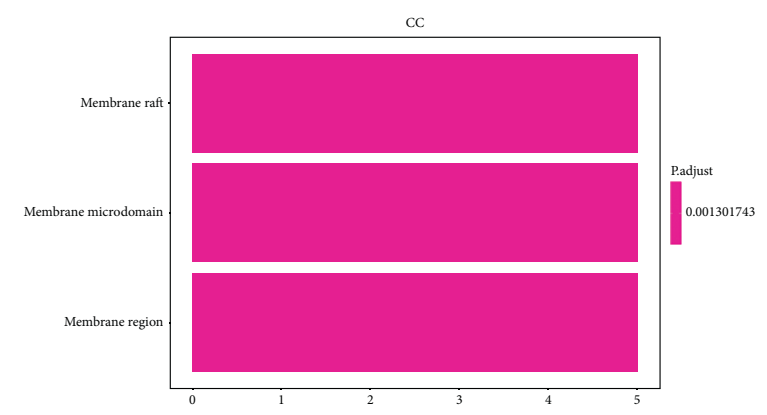

(c)

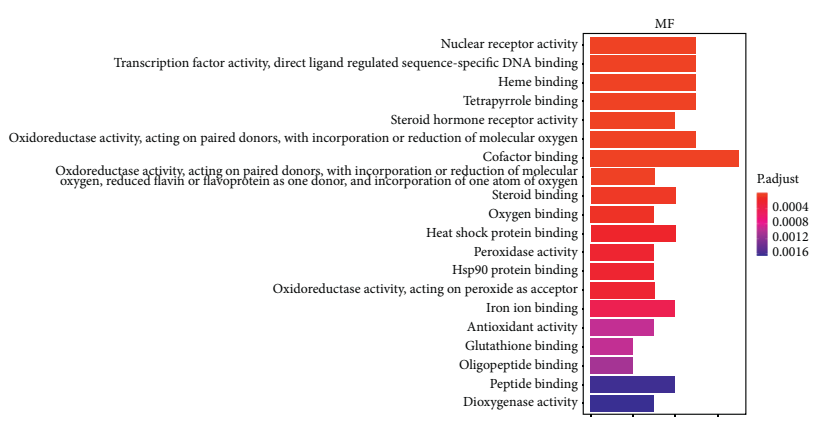

(b)

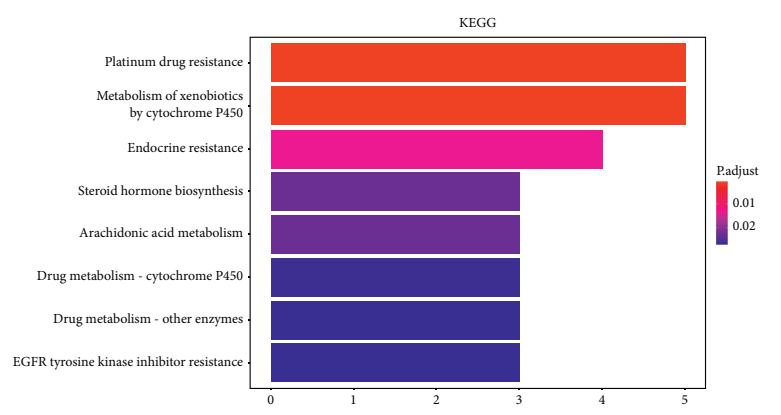

(d)

Figure 3: The GO function and KEGG pathway enrichments. (a) Enriched BP functions of active target genes; (b) enriched MF activities of active target genes; (c) enriched CC regions of active target genes; and (d) KEGG pathway enrichments.

PDM have been enriched significantly in nuclear receptor activities, transcription factor activities/direct ligand regulated sequence-specific DNA binding, heme binding, tetrapyrrole binding, steroid hormone receptor activities, oxidoreductase activities/acting on paired donors/with incorporation or reduction of molecular oxygen, cofactor binding, oxidoreductase activities/acting on paired donors/ with incorporation or reduction of molecular oxygen/reduced flavin or flavoprotein as one donor/and incorporation of one atom of oxygen, steroid binding, oxygen binding, and other functions (Supplementary File 4B). The 20 most enriched GO MFs are visualized in Figure 3(b) and ranked according to their adj. $P$ values.

The GO function enrichment analysis of CC indicated the following: the active target genes of SWD that act on PDM have been enriched significantly in membrane raft, membrane microdomain, and membrane region (Supplementary File 4C). The three most enriched GO CCs are visualized in Figure 3(c) and ranked according to their adj. $P$ values.

The KEGG pathway enrichment analysis has shown that active target genes of SWD that act on PDM were enriched significantly in platinum drug resistance, xenobiotics metabolism induced by cytochrome P450, endocrine resistance, steroid hormone biosynthesis, arachidonic acid metabolism, metabolism of drugs by cytochrome P450, metabolism of drugs by other enzymes, and epidermal growth factor receptor (EGFR) tyrosine kinase inhibitor resistance (Supplementary File 4D). The eight most enriched KEGG pathways are visualized in Figure 3(d) and ranked according to their adj $P$ values; in addition, the KEGG pathways are shown in Supplementary File 5.
By using ClueGO and CluePedia plugin apps, found in Cytoscape software, the GO function enrichment as well as KEGG pathway enrichment were visualized extra naturally (Figure 4).

3.5. Constructed Compound-Target Network and Selection of Key Compounds. We utilized Cytoscape software to create a compound-target network and the Network Analyzer tool to perform the analysis. As MOL001918, MOL001924, and MOL000433 had no correspondences to an overlapping target gene, the 23 overlapping active target genes correlated with the following 11 active compounds: 6 from RPA, 5 from RC, 2 from RAS, and 2 from RRP (Supplementary File 6A). There were 34 nodes ( 11 compound nodes as well as 23 target gene nodes) and 62 edges in total identified in the network (Supplementary File 6B, Figure 5).

The top four compounds ranked according to their degree within the network were MOL000422 (kaempferol), MOL000358 (beta-sitosterol), MOL000449 (stigmasterol), and MOL002135 (myricanone), which can be regarded as the essential compounds in SWD that act on PDM. General information of the essential compounds can be found in Table 3, and the 2D structure of these compounds acquired from the PubChem Database is shown in Figure 6.

3.6. Construction the PPI Network and Selecting Key Targets. The PPI network was obtained by importing a total of 23 overlapping active target genes into the STRING database. After setting the interaction score to a minimum value of 0.40, 23 target proteins with interactions were identified in 


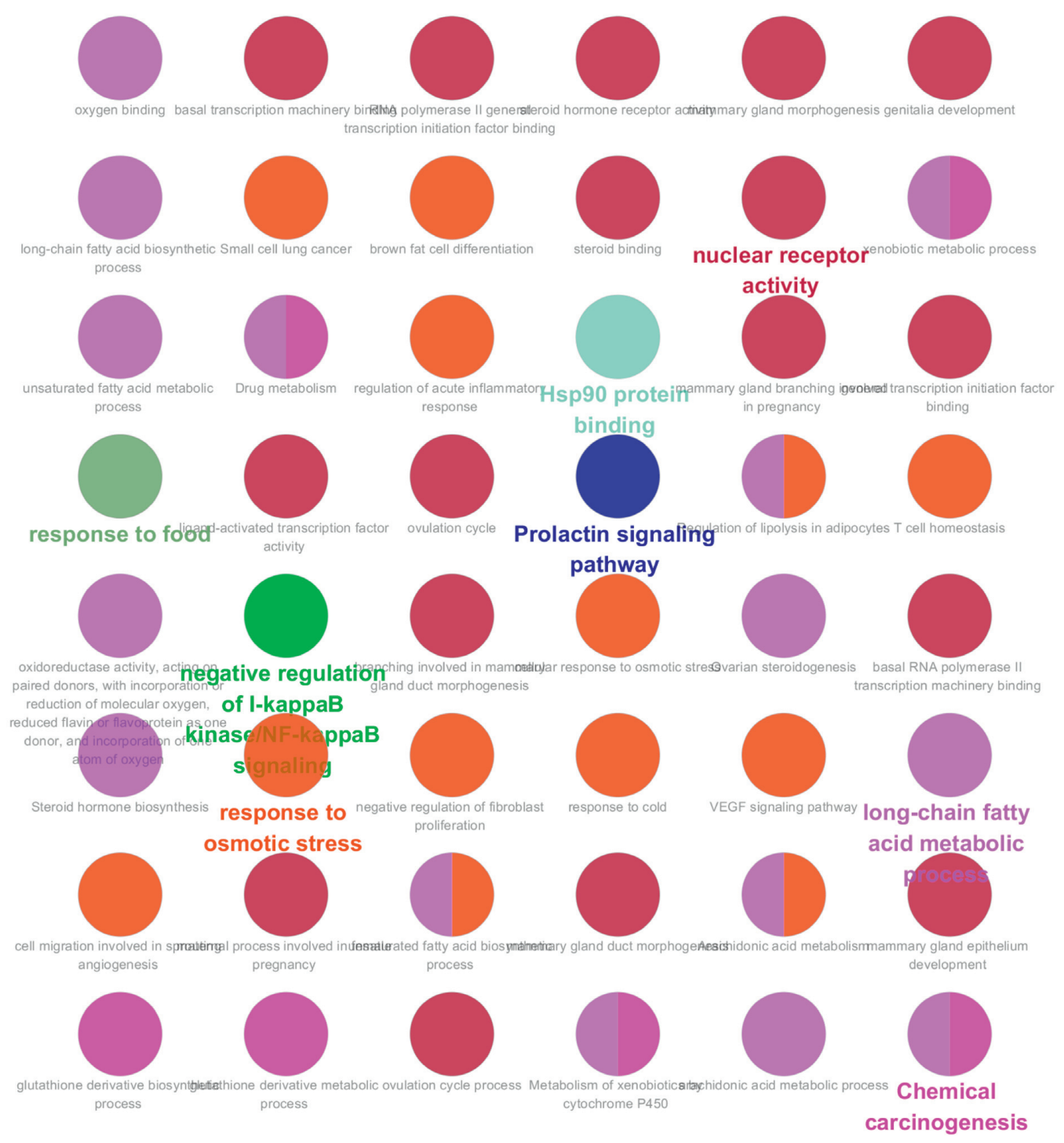

FIGURE 4: GO function enrichment and KEGG pathway enrichment visualized by ClueGO.

the network as well as 90 edges that depict the various interactions between different proteins (Supplementary File 7 and Figure 7).

The target genes that were ranked as top six in the network according to degree were selected and regarded as the key targets of SWD that act on PDM (Table 4).

3.7. Constructed Key Compound-Target Network. After introducing the key compounds (small-molecule compounds) and key targets (macromolecular protein target receptors) and their relationships to Cytoscape 3.6.0 software, a key compound-target network could be constructed (Supplementary File 8, Figure 8).

3.8. Verification Using Molecular Docking. Data on the 3D compositions of the small-molecule compounds were acquired through the PubChem Database and that of the macromolecular protein target receptors through the RCSB
PDB database. Then, AutoDockTool and AutoDock Vina software were used to perform simulations of the molecular docking of potential targets and their designated compounds. Finally, we performed molecular docking to verify the binding of target and its designated component. The simulations of the molecular docking of AKT1-kaempferol are shown in Figure 9, and those of CASP3-beta-sitosterol are shown in Figure 10. The molecular docking simulations of all of the key targets and key compounds are shown in Supplementary File 9.

\section{Discussion}

PDM is characterized by cold coagulation, qi stagnation, blood stasis, dampness and heat, and a shortage of blood and qi according to the Traditional Chinese Medicine theory, which considers a blood and qi disorder as the primary etiology of PDM and accentuates that patients should be treated in accordance to these aspects [21, 22]. According to 


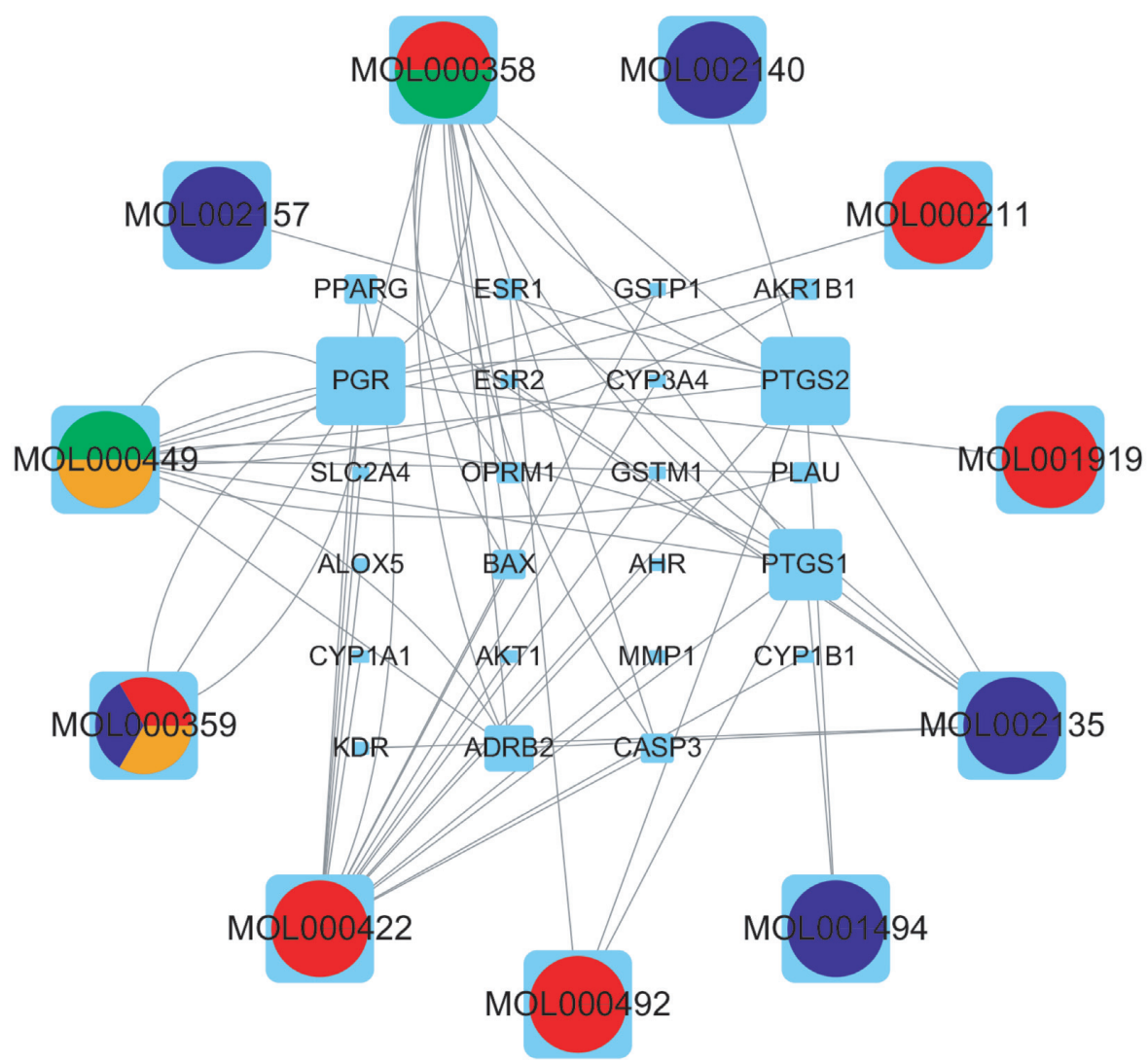

Figure 5: Compound-target network. There were 34 nodes (11 compound nodes and 23 target gene nodes) and 62 edges in the network. Circles represent active compounds (red represent compounds from RPA, bule represents compounds from RC, green represents compounds from RAS, and yellow represent compounds from RRP), rectangles represent the active target genes, and the edges represent links between the nodes.

TABLE 3: Key compounds in SWD that act on PDM.

\begin{tabular}{|c|c|c|c|c|}
\hline Compound name & PubChem CID & Molecular formula & Degree & Medicine \\
\hline Kaempferol & 5280863 & $\mathrm{C}_{15} \mathrm{H}_{10} \mathrm{O}_{6}$ & 17 & RPA \\
\hline Beta-sitosterol & 222284 & $\mathrm{C}_{29} \mathrm{H}_{50} \mathrm{O}$ & 14 & $\begin{array}{l}\text { RPA } \\
\text { RAS }\end{array}$ \\
\hline Stigmasterol & 5280794 & $\mathrm{C}_{29} \mathrm{H}_{48} \mathrm{O}$ & 12 & $\begin{array}{l}\text { RAS } \\
\text { RRP }\end{array}$ \\
\hline Myricanone & 161748 & $\mathrm{C}_{21} \mathrm{H}_{24} \mathrm{O}_{5}$ & 7 & $\mathrm{RC}$ \\
\hline
\end{tabular}

modern medicine, the pathogenesis of PDM is primarily related to prostaglandin, vasopressin, oxytocin, estradiol, and endothelin, and it is closely related to immune function and endocrine dysfunction [23, 24].

SWD has been considered as the basic prescription and the first prescription in the treatment of gynecological diseases, which consists of RPA, RC, RAS, and RRP. RPA tonifies the blood to collect yin and calms the liver to relieve pain. RC moves qi to relieve pain. RAS tonifies and harmonizes the blood and regulates menstruation to relieve pain. Finally, RRP tonifies the blood and nourishes yin $[8,9]$.

At present, there is a wide application of network pharmacology in research related to CHM. It supports the methodology of researching network-based as well as multicomponent therapies, which is compatible with the multipathway, multitarget, and multicomponent features of CHM [25, 26].

In this study, 23 active target genes of SWD that act on PDM were detected. The GO BP function enrichment analysis of SWD as PDM treatment were significantly enriched as reaction to numerous processes as described in the results section and shown in Supplementary File 4A and Figure 3(a). The GO analysis of MF showed that SWD as PDM treatment involved many different activities as described previously in the results section and Supplementary File $4 \mathrm{~B}$ and Figure 3 (b). The results of the GO CC for SWD as PDM treatment are shown in Supplementary File 4C and Figure 3(c). These results indicated that these enrichments 
<smiles>COc1ccc(-c2oc3cc(O)cc(O)c3c(=O)c2O)cc1</smiles>

(a)

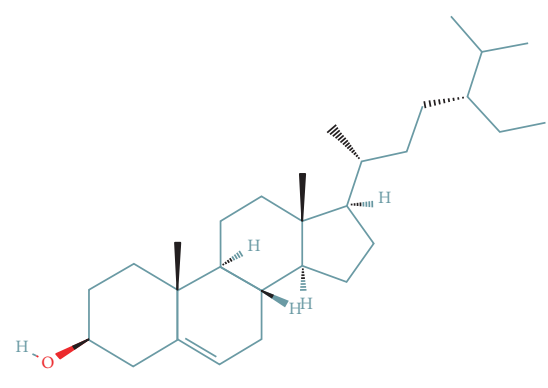

(b)

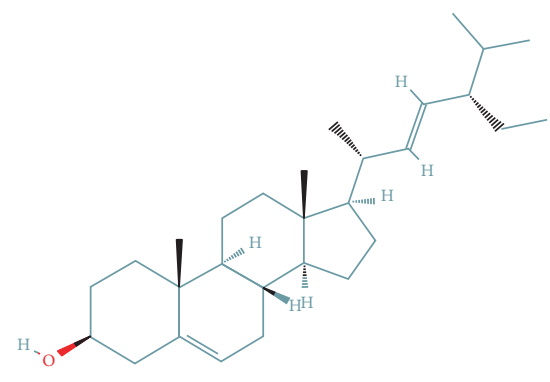

(c)<smiles>COc1c(O)cc(-c2c3ccc(O)c2Cc2ccc(c(OC)c2O)CCCCC(=O)CC3)c(O)c1O</smiles>

(d)

FIgURE 6: 2D structure of the key compounds. (a) Kaempferol; (b) beta-sitosterol; (c) stigmasterol; (d) myricanone.

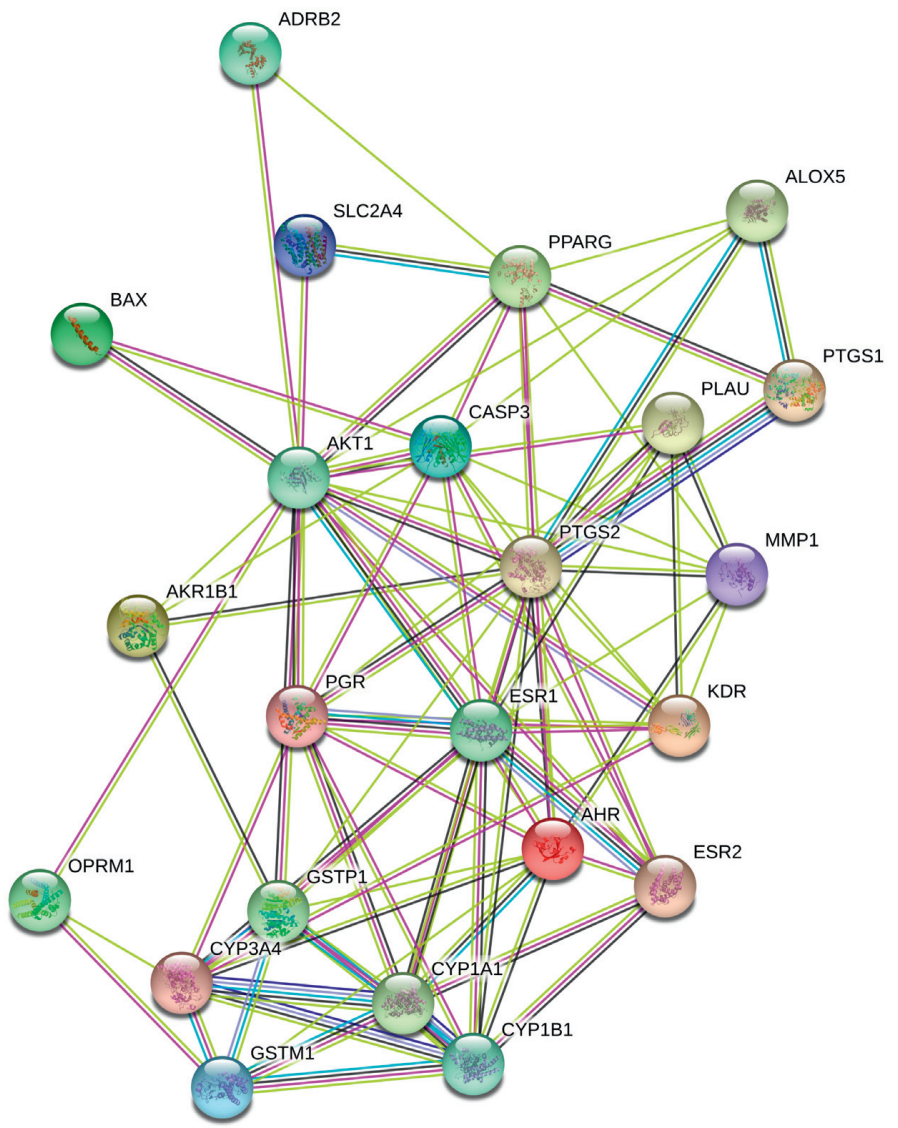

FIgURE 7: The PPI network of the SWD acting on PDM. In the network, 23 target proteins had an interaction and 90 edges represented the interactions between the proteins, when lowest interaction score was set to 0.40 . 
TABLE 4: Key targets of SWD that act on PDM.

\begin{tabular}{lcccc}
\hline Key target & Entry & Entry name & Protein names & Degree \\
\hline AKT1 & P31749 & AKT1_HUMAN & RAC-alpha serine/threonine-protein & 17 \\
PTGS2 & P35354 & PGH2_HUMAN & Prostaglandin G/H synthase 2 & 16 \\
ESR1 & P03372 & ESR1_HUMAN & Estrogen receptor & 13 \\
AHR & P35869 & AHR_HUMAN & Aryl hydrocarbon receptor & 12 \\
CASP3 & P42574 & CASP3_HUMAN & Caspase-3 & 11 \\
PGR & P06401 & PRGR_HUMAN & Progesterone receptor & 10 \\
\hline
\end{tabular}

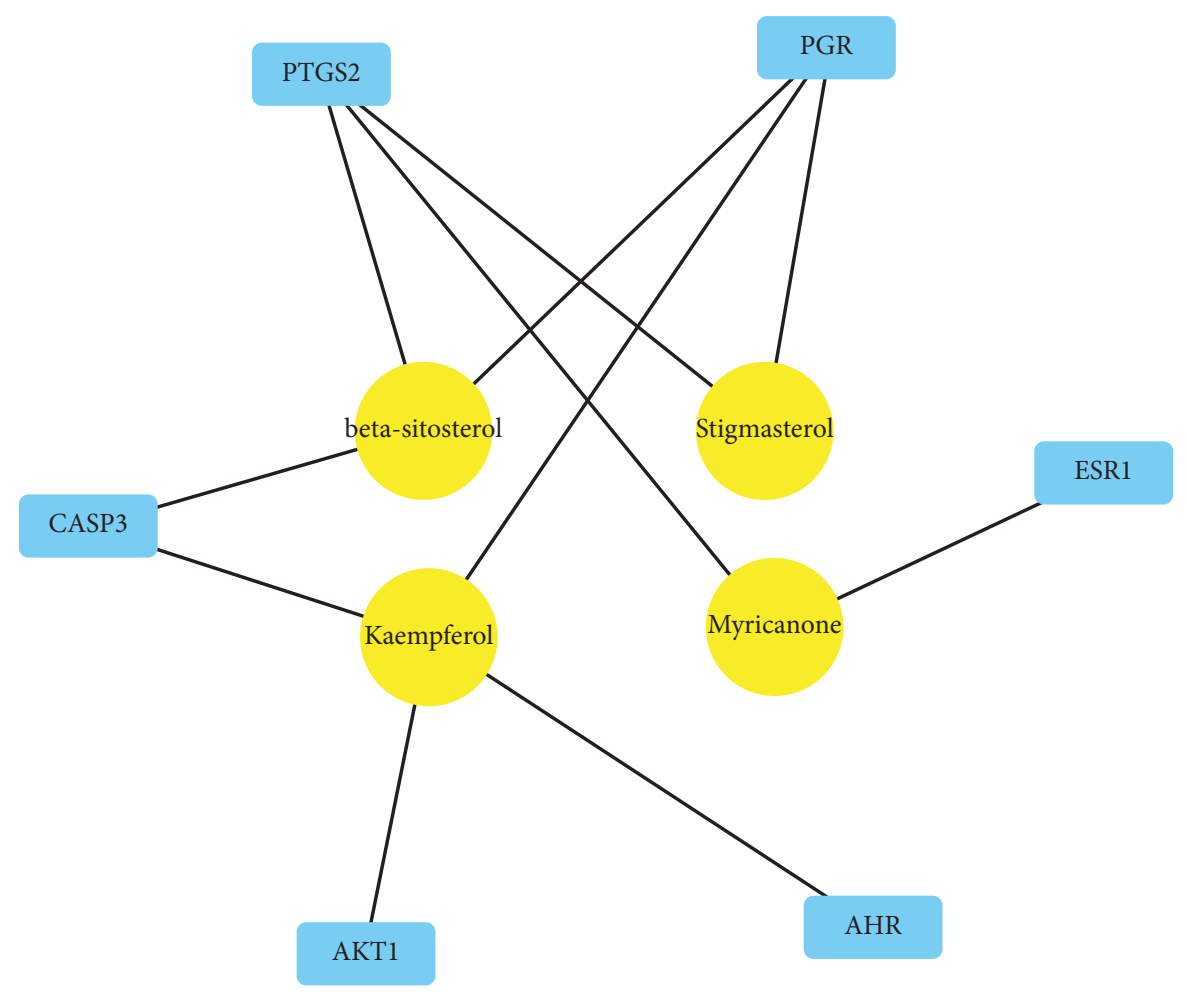

FiguRE 8: Key compound-target network. Kaempferol, beta-sitosterol, Stigmasterol, and Myricanone are the key compounds considered to be small-molecule compounds, while AKT1, PTGS2, ESR1, AHR, CASP3, and PGR are the key targets considered to be macromolecular protein target receptors.

are closely related to immunity, metabolism, and endocrine activity, which are all strongly associated to the mechanisms of disease in PDM [27-30].

The results of the KEGG enrichment pathway analysis also demonstrated that numerous pathways were strongly associated to the pathological mechanisms of PDM as shown in Supplementary Files 4D and 5 as well as Figure 3(d). These results have clearly demonstrated that SWD can act on PDM via various pathways. These pathways as well as relevant target genes are important and should be further studied.

In the present study, a network of compound-target network was created, followed by the identification of the essential compounds of SWD that act on PDM. The following essential compounds were identified: kaempferol, beta-sitosterol, stigmasterol, and myricanone. Kaempferol can be used to treat numerous acute and chronic inflammation-induced diseases due to its anti-inflammatory properties [31]. Beta-sitosterol has anti-inflammatory, anthelminthic, and antimutagenic activities [32].
Stigmasterol is an organic steroid alcohol that possesses the well-known ability of immune modulation [33]. Myricanone has been reported to have apoptosis-promoting abilities [34]. These studies were related to the regulation of immunity and endocrine function. The SWD action on PDM could be the result of the interaction of these multiple compounds. However, there are few related studies regarding the single compound action on PDM, which requires further investigation.

The PPI network showed that the actions of SWD on PDM were associated with various targets. Of which, the most essential targets were AKT1, PTGS2, ESR1, AHR, CASP3, and PGR. Previous research indicated that the levels of PTGS2 and ESR1 were abnormal in an estradiol benzoateoxytocin induced dysmenorrhea mice model [9]. It has been shown that the ESR1 gene is statistically significant different in PDM patients and controls [35]. The polymorphisms PGR-CYP17A1-CYP19A1 have been shown to have a genegene interaction when there is a risk of developing 


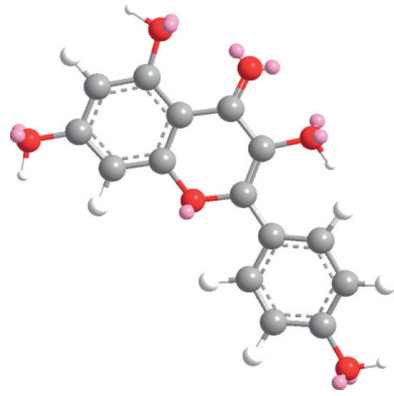

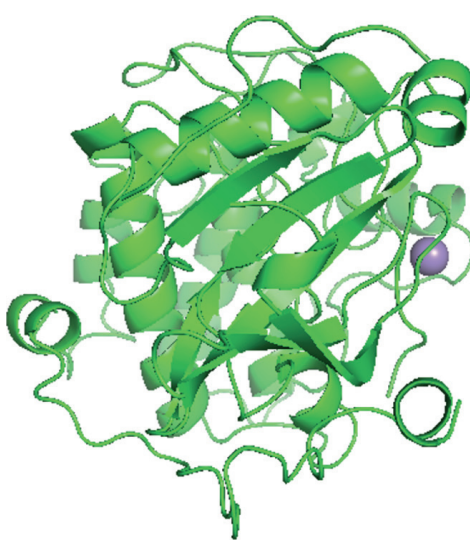

(b)

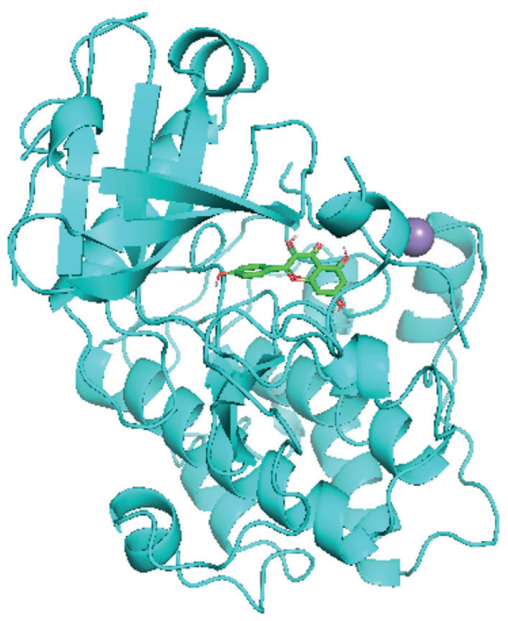

(c) (a)

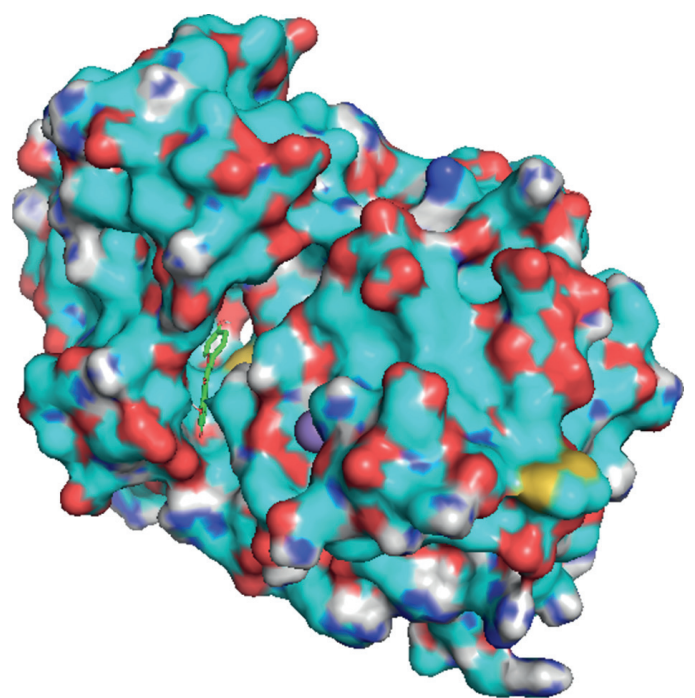

(d)

Figure 9: AKT1 kaempferol molecular docking. 3D structures of (a) kaempferol and (b) 3D structures of AKT1; (c) molecular docking simulation; and (d) molecular docking simulation (display protein surface).

endometriosis [36]. The relationship between these genes and PDM, some of which already have relevant studies, can be further studied, and the possible mechanisms can be explored, which have not previously been investigated.

Molecular docking was also conducted to identify specific interactions among key compounds and their predicted protein targets, which could improve the network's accuracy [37]. The preliminary molecular docking results showed kaempferol, beta-sitosterol, stigmasterol, and myricanone, the key active compounds in RPA, RC, RAS, and RRP, had high binding activities with AKT1, PTGS2, ESR1, AHR, CASP3, and PGR gene target proteins. These active compounds may be important foundational materials in SWD for treating PDM through related signaling pathways.

A network pharmacology approach was adopted with the intention of studying the various pharmacological mechanisms of SWD that act on PDM, and the binding of the target and its corresponding compound were verified using molecular docking. However, there are some limitations using these approaches. First, we used the TCMSP database to detect the active compounds of SWD and their corresponding target genes. The criteria for screening the active compounds were fixed, and the target genes associated with PDM were acquired through the GeneCards database. Even though at present these databases are increasingly comprehensive, some compounds and target genes may still have been omitted. Second, the GO function enrichment and KEGG pathway enrichment analyses were undertaken, in addition to the construction of a PPI network to investigate the pathways and target genes of the SWD acting on PDM. These potential target genes and pathways require further study using experimental analyses. Third, preliminary molecular docking verification was only conducted in this study, and research regarding the molecular docking of small-molecule compounds and macromolecular protein target receptors should be further studied. 


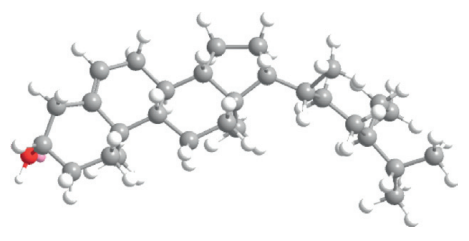

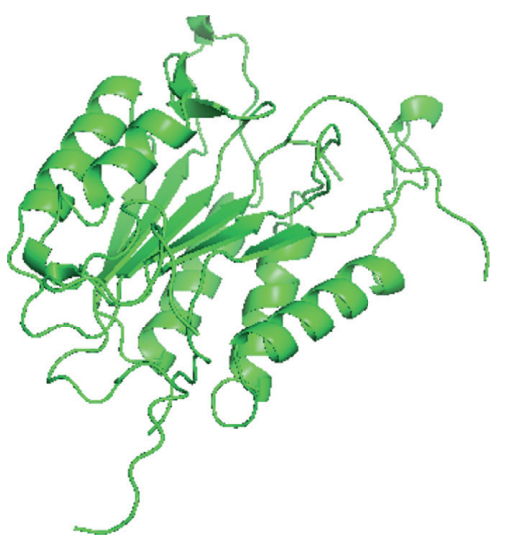

(b)

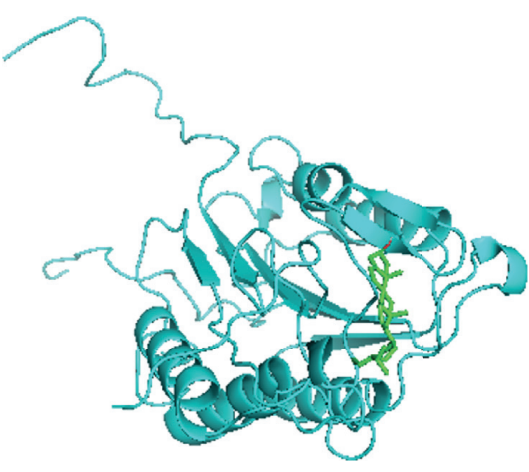

(c) (a)

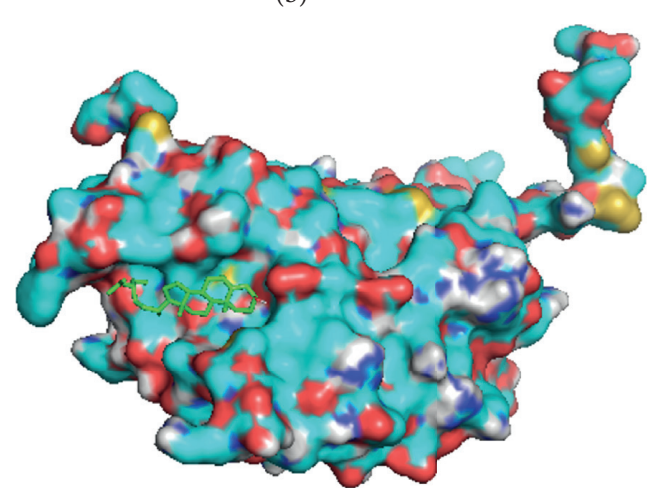

(d)

FIGURE 10: CASP3 beta-sitosterol molecular docking. 3D structures of (a) beta-sitosterol and (b) CASP3; (c) molecular docking simulation; and (d) molecular docking simulation (display protein surface).

\section{Conclusion}

In conclusion, the interactions among the active compounds, pathways, and target genes of SWD acting on PDM, via the effects of various compounds, targets, and pathways, were investigated in the present study. In addition, molecular docking was conducted lastly to identify specific interactions between key compounds and their predicted target proteins, which indicated these compounds may be an important material foundation in the SWD for treating PDM. In sum, the pharmacological mechanisms of SWD that act on PDM were investigated, and the active compounds in the SWD for treating PDM were further verified.

\section{Data Availability}

The data used in this study can be found online as supplementary files (http://www.mediafire.com/file/ 87m1y65c596wxbt/Supplementary_Files.zip/file).

\section{Conflicts of Interest}

The authors declare that they have no conflicts of interest.

\section{Authors' Contributions}

Dandan Jiang and Xiaoyan Wang created and designed this study and wrote the manuscript. Dandan Jiang and Yufeng
Zhang collated and extracted data. Dandan Jiang, Lijun Tian, and Yufeng Zhang conducted analysis of the data. Lijun Tian and Yufeng Zhang supervised and conducted project administration. All the authors have read and authorized the final version of this paper.

\section{Acknowledgments}

First, we would like to acknowledge the software and platform that provided the bioinformatic tools. Second, this study was supported in part by the Nantong Science and Technology Plan Project (grant MS12017004-2, recipient Tian) as well as the Research Grant of Jiangyin Hospital of Traditional Chinese Medicine (recipient Zhang). Lastly, we would like to thank LetPub (http://www.letpub.com) for its linguistic guidance while preparing this paper.

\section{Supplementary Materials}

Supplementary files can be found online (). Supplementary File 1: compounds of SWD from TCMSP: (A) 86 compounds of RPA from TCMSP; (B) 189 compounds of RC from TCMSP; (C) 125 compounds of RAS from TCMSP; (D) 76 compounds of RRP from TCMSP. Supplementary File 2: target genes of SWD: (A) corresponding target genes of active compounds; (B) corresponding target gene symbols of active compounds; (C) 97 active target genes of SWD. 
Supplementary File 3 PDM-related target genes. (A) PDMrelated target genes from GeneCards; (B) 299 PDM-related target genes. Supplementary File 4: GO function enrichment and KEGG pathway enrichment analysis: (A) BP function enrichment analysis; (B) MF function enrichment analysis; (C) CC function enrichment analysis; (D) KEGG pathway enrichment analysis. Supplementary File 5: KEGG pathway. Supplementary File 6: compound-target network: (A) compounds and targets of compound-target network; (B) data of compound-target network. Supplementary File 7: data of PPI network. Supplementary File 8: data of key compound-target network. Supplementary File 9: molecular docking simulations of all the key targets and key compounds. (Supplementary Materials)

\section{References}

[1] K. A. Kho and J. K. Shields, "Diagnosis and management of primary dysmenorrhea," JAMA, vol. 323, no. 3, pp. 268-269, 2020.

[2] V. De Sanctis, A. Soliman, S. Bernasconi et al., "Primary dysmenorrhea in adolescents: prevalence, impact and recent knowledge," Pediatric Endocrinology Reviews, vol. 13, no. 2, pp. 512-520, 2015.

[3] I. Guimarães and A. M. Póvoa, "Primary dysmenorrhea: assessment and treatment," Revista Brasileira de Ginecologia e Obstetrícia/RBGO Gynecology and Obstetrics, vol. 42, no. 08, pp. 501-507, 2020.

[4] G. Grandi, A. Napolitano, A. Xholli, A. Tirelli, C. Di Carlo, and A. Cagnacci, "Effect of oral contraceptives containing estradiol and nomegestrol acetate or ethinyl-estradiol and chlormadinone acetate on primary dysmenorrhea," Gynecological Endocrinology, vol. 31, no. 10, pp. 774-778, 2015.

[5] L. Gao, C. Jia, H. Zhang, and C. Ma, "Wenjing decoction (herbal medicine) for the treatment of primary dysmenorrhea: a systematic review and meta-analysis," Archives of Gynecology and Obstetrics, vol. 296, no. 4, pp. 679-689, 2017.

[6] L. Gao, Z. Xiao, C. Jia, and W. Wang, "A comparison of the efficacy of Chinese herbal medicines in the treatment of primary dysmenorrhea: a network meta-analysis protocol," Medicine (Baltimore), vol. 98, no. 14, Article ID e15100, 2019.

[7] C. Chai, F. Hong, Y. Yan et al., "Effect of traditional Chinese medicine formula GeGen decoction on primary dysmenorrhea: a randomized controlled trial study," Journal of Ethnopharmacology, vol. 261, Article ID 113053, 2020.

[8] J. Li, J. F. Shi, X. Nie, H. J. Jiang, C. M. Fu, and Y. He, "[Network pharmacology-based study on mechanism of Siwu Decoction in treatment of primary dysmenorrhea]," Zhongguo Zhong Yao Za Zhi, vol. 44, no. 3, pp. 559-565, 2019.

[9] J. Li, H. J. Jiang, Y. He et al., "[Prescription compatibility connotation and action mechanism of Siwu Decoction in treating primary dysmenorrhea]," Zhongguo Zhong Yao Za Zhi, vol. 45, no. 12, pp. 2947-2953, 2020.

[10] G. Li, A. Liu, M. Lin, S. Liao, and Z. Wen, "Chinese herbal formula siwutang for treating primary dysmenorrhea: a systematic review and meta-analysis of randomized controlled trials," Maturitas, vol. 138, pp. 26-35, 2020.

[11] J. Ru, P. Li, J. Wang et al., "TCMSP: a database of systems pharmacology for drug discovery from herbal medicines," Journal of Cheminformatics, vol. 6, p. 13, 2014.

[12] Q. Xia, M. Liu, H. Li, L. Tian, J. Qi, and Y. Zhang, "Network pharmacology strategy to investigate the pharmacological mechanism of HuangQiXiXin decoction on cough variant asthma and evidence-based medicine approach validation," Evidence-based Complementary and Alternative Medicine, vol. 2020, Article ID 3829092, 15 pages, 2020.

[13] C. T. UniProt, "UniProt: the universal protein knowledgebase," Nucleic Acids Research, vol. 46, no. 5, p. 2699, 2018.

[14] G. Stelzer, N. Rosen, I. Plaschkes et al., "The GeneCards suite: from gene data mining to disease genome sequence analyses," Current Protocols in Bioinformatics, vol. 54, pp. 1-30, 2016.

[15] G. Yu, L.-G. Wang, Y. Han, and Q.-Y. He, "clusterProfiler: an $\mathrm{R}$ package for comparing biological themes among gene clusters," OMICS: A Journal of Integrative Biology, vol. 16, no. 5, pp. 284-287, 2012.

[16] P. Shannon, A. Markiel, O. Ozier et al., "Cytoscape: a software environment for integrated models of biomolecular interaction networks," Genome Research, vol. 13, no. 11, pp. 2498-2504, 2003.

[17] G. Bindea, J. Galon, and B. Mlecnik, "CluePedia Cytoscape plugin: pathway insights using integrated experimental and in silico data," Bioinformatics, vol. 29, no. 5, pp. 661-663, 2013.

[18] G. Bindea, B. Mlecnik, H. Hackl et al., "ClueGO: a Cytoscape plug-in to decipher functionally grouped gene ontology and pathway annotation networks," Bioinformatics, vol. 25, no. 8, pp. 1091-1093, 2009.

[19] D. Szklarczyk, A. L. Gable, D. Lyon et al., "STRING v11: protein-protein association networks with increased coverage, supporting functional discovery in genome-wide experimental datasets," Nucleic Acids Research, vol. 47, no. D1, pp. D607-D613, 2019.

[20] M. F. Sanner, "Python: a programming language for software integration and development," Journal of Molecular Graphics \& Modelling, vol. 17, no. 1, pp. 57-61, 1999.

[21] G. B. Shetty, B. Shetty, and A. Mooventhan, "Efficacy of acupuncture in the management of primary dysmenorrhea: a randomized controlled trial," Journal of Acupuncture and Meridian Studies, vol. 11, no. 4, pp. 153-158, 2018.

[22] S.-D. Kim, "Yoga for menstrual pain in primary dysmenorrhea: a meta-analysis of randomized controlled trials," Complementary Therapies in Clinical Practice, vol. 36, pp. 94-99, 2019.

[23] R. Liedman, S. R. Hansson, D. Howe et al., "Reproductive hormones in plasma over the menstrual cycle in primary dysmenorrhea compared with healthy subjects," Gynecological Endocrinology, vol. 24, no. 9, pp. 508-513, 2008.

[24] L. Fang, C. Gu, X. Liu et al., "Metabolomics study on primary dysmenorrhea patients during the luteal regression stage based on ultra performance liquid chromatography coupled with quadrupole-time-of-flight mass spectrometry," Molecular Medicine Reports, vol. 15, no. 3, pp. 1043-1050, 2017.

[25] R. Zhang, X. Zhu, H. Bai, and K. Ning, "Network pharmacology databases for traditional Chinese medicine: review and assessment," Frontiers in Pharmacology, vol. 10, p. 123, 2019.

[26] Y. Zhang, W. Jiang, Q. Xia, J. Qi, and M. Cao, "Pharmacological mechanism of Astragalus and Angelica in the treatment of idiopathic pulmonary fibrosis based on network pharmacology," European Journal of Integrative Medicine, vol. 32, Article ID 101003, 2019.

[27] Q. Zhang, S. Yu, Y. Wang et al., “Abnormal reward system network in primary dysmenorrhea," Molecular Pain, vol. 15, Article ID 2069301424, 2019.

[28] P. Kannan, K.-K. Cheung, and B. W.-M. Lau, "Does aerobic exercise induced-analgesia occur through hormone and inflammatory cytokine-mediated mechanisms in primary dysmenorrhea?" Medical Hypotheses, vol. 123, pp. 50-54, 2019.

[29] Y. X. Ma, X. Y. Yang, G. Guo, D. Q. Du, Y. P. Yu, and S. Z. Gao, "Research of herb-partitioned moxibustion for 
primary dysmenorrhea patients based on the LC-MS metabonomics," Evidence-Based Complementary and Alternative Medicine, vol. 2015, Article ID 621490, , 2015.

[30] P. Liu, J. Duan, P. Wang et al., "Biomarkers of primary dysmenorrhea and herbal formula intervention: an exploratory metabonomics study of blood plasma and urine," Molecular Omics, vol. 9, no. 1, pp. 77-87, 2013.

[31] J. Ren, Y. Lu, Y. Qian, B. Chen, T. Wu, and G. Ji, "Recent progress regarding kaempferol for the treatment of various diseases," Experimental and Therapeutic Medicine, vol. 18, no. 4, pp. 2759-2776, 2019.

[32] I. M. Villaseñor, J. Angelada, A. P. Canlas, and D. Echegoyen, "Bioactivity studies on $\beta$-sitosterol and its glucoside," Phytotherapy Research, vol. 16, no. 5, pp. 417-421, 2002.

[33] A. O. Antwi, D. D. Obiri, N. Osafo, L. B. Essel, A. D. Forkuo, and C. Atobiga, "Stigmasterol alleviates cutaneous allergic responses in rodents," Biomed Research International, vol. 2018, Article ID 3984068, 13 pages, 2018.

[34] A. Paul, J. Das, S. Das, A. Samadder, and A. R. Khuda-Bukhsh, "Anticancer potential of myricanone, a major bioactive component of Myrica cerifera: novel signaling cascade for accomplishing apoptosis," Journal of Acupuncture and Meridian Studies, vol. 6, no. 4, pp. 188-198, 2013.

[35] A. Z. Ozsoy, N. Karakus, S. Yigit, B. Cakmak, M. C. Nacar, and H. Yilmaz Dogru, "The evaluation oflL6andESR1Gene polymorphisms in primary dysmenorrhea," Immunological Investigations, vol. 45, no. 1, pp. 75-86, 2016.

[36] J. Cardoso, D. Machado, R. Ferrari, M. Silva, P. Berardo, and J. Perini, "Combined effect of the PGR +331C > T, CYP17A1 $-34 \mathrm{~A}>\mathrm{G}$ and CYP19A1 1531G > A polymorphisms on the risk of developing endometriosis," Revista Brasileira de Ginecologia e Obstetrícia/RBGO Gynecology and Obstetrics, vol. 39, no. 06, pp. 273-281, 2017.

[37] P. Yi, Z. Zhang, S. Huang, J. Huang, W. Peng, and J. Yang, "Integrated meta-analysis, network pharmacology, and molecular docking to investigate the efficacy and potential pharmacological mechanism of Kai-Xin-San on Alzheimer's disease," Pharmaceutical Biology, vol. 58, no. 1, pp. 932-943, 2020 . 